\title{
The duration effects of lumbar extensor muscle fatigue on postural control in soccer players
}

\author{
Mehdi Ahmadi ${ }^{1}$, Afsun Nodehi Moghadam ${ }^{1 *}$, Majid Bakhshi ${ }^{2}$, Tahere Rezaeian ${ }^{1}$, \\ Gholamreza Pahnabi ${ }^{3}$
}

Received: 2 Sep 2016

Published: 20 Dec 2017

\begin{abstract}
Background: The ability to maintain body position in space is called postural control. Muscular fatigue created after doing voluntary repeated contractions impairs postural control. Thus, the present study was conducted to compare the effects of fatigue of the lumbar extensor muscles and duration of these effects on postural sway between soccer player and non-athlete participants.

Methods: A total of 15 male soccer players and 15 male non-athletes participated in this cross- sectional study. Lumbar extensor fatigue is produced by a dynamometer and maintains $60 \%$ of the maximum voluntary isometric contraction in standing position. The mean velocity and the total area of the participants were evaluated using force plate at baseline, immediately, 3,6 , and 9 minutes after fatigue and compared between the 2 groups. Descriptive statistics, repeated measurement ANOVA, and independent $t$ test were used for data analysis.

Results: The results demonstrated that fatigue of the waist extensor muscles affects the postural sway ( $\mathrm{p}=0.001)$. Also, the total area level was recovered earlier than the mean velocity after fatigue. The results revealed that total area and velocity with eyes closed decreased in the soccer player group compared with the non-athlete group.

Conclusion: Lumbar extensors fatigue significantly changed the mean velocity and the total area in soccer players and non-athletes. Lumbar fatigue had more effects on the total area than the mean velocity in the two groups.
\end{abstract}

Keywords: Muscle fatigue, Postural control, Balance, Lumbar, Athlete

Copyright $\odot$ Iran University of Medical Sciences

Cite this article as: Ahmadi M, Nodehi Moghadam A, Rajabzadeh A, Rezaeian T, Bakhshi M, Pahnabi Gh. The duration effects of lumbar extensor muscle fatigue on postural control in soccer players. Med J Islam Repub Iran. 2017 (20 Dec);31:112. https://doi.org/10.14196/mjiri.31.112

\section{Introduction}

The ability to maintain body position in space is called postural control that it depends on stability and orientation (1). Postural stability refers to the ability to maintain body balance. Based on evidence, the cooperation of sensory systems (visual, proprioception, and vestibular system), motor system, and central nervous system are needed to maintain postural control (2). Body joints act as a steady chain and disturbance in any muscle impairs body balance (3). Moreover, muscular fatigue after doing voluntarily repeated contractions impairs postural control (4). Muscular fatigue refers to muscles' inability to produce the expected power (5). Rampinini found a relationship between central fatigue indicators and maximal voluntary contraction (MVC). Also,

Corresponding author: Afsun Nodehi Moghadam, afsoonnodehi@gmail.com

1. Department of Physical Therapy, School of Medicine, University of Social Welfare and Rehabilitation Sciences, Tehran, Iran,

2. Department of Corrective Exercises and Pathology, University of Shahrekord, Shahrekord, Iran.

3. Department of Physical Therapy, School of Medicine, Tehran University of Medical Sciences, Tehran, Iran. sprint performance will decrease, whereas muscle soreness occurs due to peripheral fatigue indicators (6). Fatigued soccer players showed decreased function of postural-control. Participants with better function in the repeated sprint ability test appeared to be less influenced by postural control impairment, especially in single-legged position (7). In addition, a football match can cause decreased postural control and performance of the lower extremities in younger players (8); 20 minutes of performance activity may have a negative effect on dynamic balance, and recovery occurs within 10 minutes after exercise in soccer players. Moreover, the level of exertion measured by resistive progressive exercise would correspond to athletes' ability

$\uparrow$ What is "already known” in this topic:

Muscular fatigue refers to muscles' inability to produce the expected power. Fatigued soccer players showed decreased function of postural control. Any disorder in the postural control increases the risk of fall and injury.

\section{$\rightarrow$ What this article adds:}

This study found and gathered the most important effects of the fatigue of the lumbar extensor muscles and duration of these effects on postural sway in soccer players and non-athlete participants to avoid further injury. 
to control their center of mass (9).

Any disorder in the postural control increases the risk of falling and injury (10); larger L5/S1 joint forces were observed during both motions after fatigue. Such changes showed potentially increased risk of back injury (9). Thus, we should increase our knowledge on the potential negative effects of acute muscle fatigue on the balance and effect of muscle endurance training as a fall risk intervention (11). The duration of muscular fatigue on balance is not exactly clear. Yaggie et al. (12) concluded that impairment in the postural control after isokinetic fatigue of ankle muscles continues for 20 minutes. Harkins et al. stated that after isokinetic fatigue of ankle muscles, there was more reduction in the power of muscles and disorder duration of the postural control (13). Nurtekinrt et al. found that the fatigue effect of aerobic remains on the balance for 13 minutes after exercising (15). Thus, we are fairly aware of the duration of fatigue effect resulting from isokinetic exercises and also basic exercises. On the other hand, Sahlin et al. conducted a study to investigate the fatigue arising from isometric contraction of the extensor muscles in the knee (16) and concluded that after isometric fatigue of knee extensor muscles, the maximum voluntary contraction and the amount of endurance in knee extensor muscles (time to be exhausted) reached to the state of before fatigue after 2 minutes and 2 to 4 minutes after fatigue, respectively. To our knowledge, to date, no study has been conducted on the duration of isometric fatigue on postural sways. With regards to the static tasks of body muscles during daily works and fatigability of these muscles, it is necessary to gain knowledge about the duration of isometric fatigue in these muscles. Outputs at the antigravity muscles are more important than other muscles (17). Thus, the present study was conducted to evaluate the effects of lumbar fatigue on the mean velocity $(\mathrm{cm} / \mathrm{s})$ and the total area $\left(\mathrm{cm}^{2}\right)$ of soccer players and non-athlete participants and to compare the duration of these effects between the 2 groups.

\section{Methods}

\section{Participants}

According to the previous studies and simple random sampling, 15 male soccer players, with a mean \pm SD age of $22.6 \pm 3.1$ years, mean \pm SD height of $175.2 \pm 4.9 \mathrm{~cm}$, and mean \pm SD BMI of $78 \pm 3.8 \mathrm{~kg}$ (Group 1) and 15 male nonathletes, with a mean \pm SD age of $24.3 \pm 2.3$ years, mean \pm SD height of $177.3 \pm 5 \mathrm{~cm}$, and mean \pm SD weight of $75 \pm 5.5 \mathrm{~kg}$ (Group 2) participated in this cross- sectional study. Informed consent was obtained from all participants. Those with history of severe trauma, musculoskeletal surgery, nervous and vestibular system impairments, and congenital anomalies were excluded from the study.

\section{Fatigue protocol}

Muscle fatigue was produced by maintenance of $60 \%$ of maximal voluntary isometric contraction (MVIC) of the lumbar extensors in this study. First, MVIC of the lumbar extensors was assessed by dynamometer (Commander Power Track II HHD. JTech Medical, USA). It was performed in standing position with $30^{\circ}$ flexion of the lumbar region. For this purpose, a device was designed (Fig. 1)

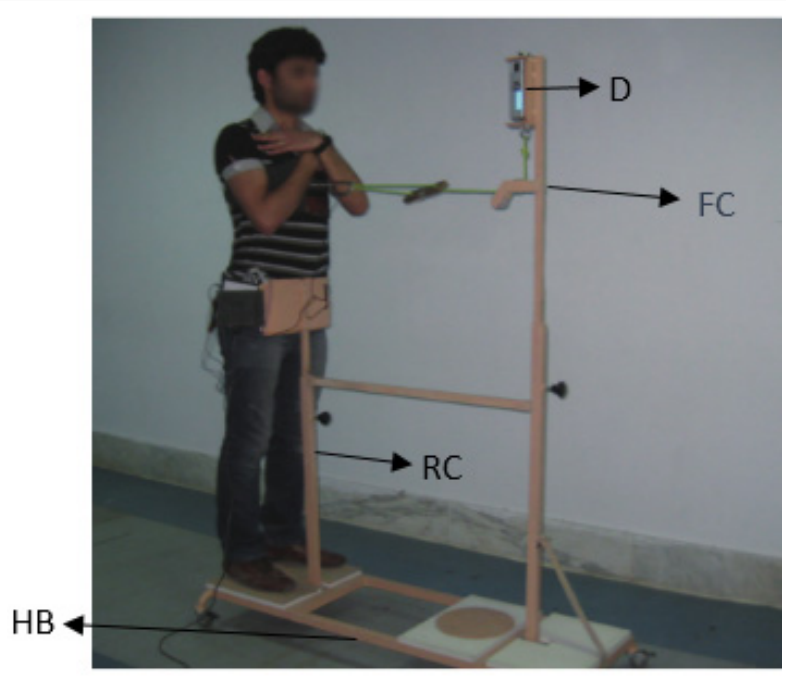

Fig. 1. FC: Front column, RC: Rear Column, D: Dynamometer, HB: Horizontal Base

with 2 vertical columns and a horizontal base, and the vertical columns could be adjusted to the height of the participants. Dynamometer was positioned at the front column. Participants wore a vest, and lumbar muscle tension transferred to the dynamometer by a rope that was attached to the front of the vest. Participants were against the rear vertical column (Fig. 1). After doing a brief warm-up, the participants were asked to exert maximum extension contraction and maintain it for 5 seconds. Continuous verbal encouragement was provided during the test. The measurement was repeated 3 times with an interval of 1 minute between each repetition. The highest value of the 3 repetitions was considered as the MVIC of the lumbar extensors. To produce muscular fatigue, after a short recovery period, the participants were asked to exert $60 \%$ of their MVIC of lumbar extensor in the same position of MVIC measurement, and maintain it until they could no longer sustain $20 \%$ of the submaximal MVIC force output (18).

\section{Measurements}

To conduct this study, each participant was evaluated in a session. Firstly, the mean velocity and total area of the participants were recorded with closed eyes for 30 seconds before fatigue using force plate. Furthermore, sways were recorded in the participants in standing position on both legs, head in neutral, feet together, hands near the body, and eyes closed immediately, 3, 6, and 9 minutes after fatigue and results were compared between the 2 groups.

Postural sway parameters including sway area and mean velocity were recorded by force platform (Kistler 9260AA6) 4th order, $10 \mathrm{~Hz}$ low-pass Butterworth filter. Signals from the force platform were sampled at $400 \mathrm{~Hz}$, amplified, and converted from analog to digital form through an A/D converter. Participants stood on the force plate comfortably on 2 limbs, head in neutral position, feet together, arms at side of the body, and eyes closed for 30 seconds. Foot placement on the force plate was marked by signs. To convert the force plate data to a numerical form, we used a formula suggested in previous studies $(19,20)$. 


\section{Statistical analysis}

Data were analyzed using SPSS16. Repeated measurement, ANOVA with repeated measurement, and independent $t$ test were used for data analysis. Post-hoc test was also applied when necessary. Significance level was set at 0.05 .

\section{Results}

All participants were able to stand 30 seconds during the test. No significant differences were obtained between the 2 groups in the anthropometric data (Table 1).

\section{Fatigue and postural control}

We used repeated measurement ANOVA to examine the effect of time (pre, post, and follow-up) on each group separately and at eyes closed condition. Also, we used repeated measurement to evaluate the effect of time (pre, post, and follow-up) on between group differences over time. The repeated measurement for between group differences was done and the results indicated that both groups were significantly different $(p=0.001)$. Also, the differences of groups changed over time $(\mathrm{p}=0.001)$ (Table 2$)$. The results revealed that total area with eyes closed $(\mathrm{p}<0.001)$ and velocity $(\mathrm{p}<0.001)$ were significantly different between the 2 groups immediately and 3 minutes after fatigue $(p=0.001)$ (Fig. 2).

Bonferroni Post Hoc results showed that total area and velocity were significantly decreased in the soccer player group compared to the non-athlete group 6 minutes after fatigue as compared to immediately $(\mathrm{p}<0.001), 9$ minutes after fatigue as compared to immediately $(\mathrm{p}<0.001), 6$ minutes after fatigue as compared to 3 minutes $(\mathrm{p}<0.001)$, and 9 minutes after fatigue as compared to 3 minutes after fatigue $(\mathrm{p}<0.001)$ (Fig. 3).

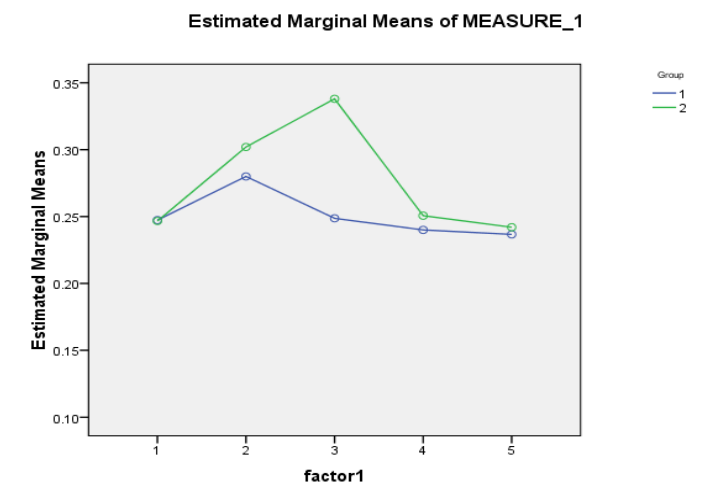

Fig. 2. Postural sway (total area) between the 2 groups (Group1: soccer players; Group2: non-athletes)

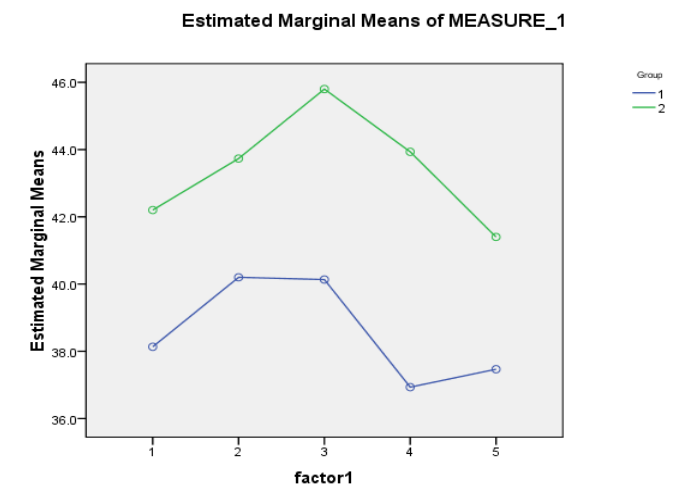

Fig. 3. Postural sway (velocity) between the 2 groups (Group1: soccer players; Group 2: non-athletes)

The results of independent $t$ test for between group differences revealed that total area with eyes closed significantly decreased in the intervention group compared to the control group immediately $(\mathrm{p}=0.001)$ and 3 minutes after fatigue $(\mathrm{p}<0.001)$ (Table 3$)$.

Table 1. Discriptive statistics of demographic information for all test subjects

\begin{tabular}{lcc}
\hline Variable & Soccer player $(\mathrm{n}=15)$ & Non-athlete $(\mathrm{n}=15)$ \\
\hline Age & $22.6 \pm 3.1$ & $24.3 \pm 2.3$ \\
Height $(\mathrm{cm})$. & $175.2 \pm 4.9$ & $177.3 \pm 5$ \\
Weight $(\mathrm{kg})$. & $78 \pm 3.8$ & $75 \pm 5.5$ \\
BMI $\left(\mathrm{kg} / \mathrm{m}^{2}\right)$ & 25.4 & 23.9 \\
\hline
\end{tabular}

Table 2. The Comparison of total area and velocity between athlete and non-athlete groups before, immediately, $3 \mathrm{~min}, 6 \mathrm{~min}$ and $9 \mathrm{~min}$ after fatigue over time (Repeated measurement analysis)

\begin{tabular}{|c|c|c|c|c|}
\hline Variables & & Athlete & Non-athlete & $\mathrm{p}^{\#}$ (group) \\
\hline \multirow[t]{6}{*}{ COP displacement $(\mathrm{cm})$ Eye Closed } & Before & $0.247 \pm 0.019$ & $0.24 \pm 0.013$ & $<0.001^{*}$ \\
\hline & Immediate & $0.28 \pm 0.018$ & $0.30 \pm 0.014$ & \\
\hline & 3 min After & $0.24 \pm 0.019$ & $0.33 \pm 0.017$ & \\
\hline & $6 \mathrm{~min}$ After & $0.24 \pm 0.019$ & $0.25 \pm 0.018$ & \\
\hline & $9 \mathrm{~min}$ After & $0.23 \pm 0.013$ & $0.24 \pm 0.016$ & \\
\hline & Before & $38.13 \pm 1.8$ & $42.2 \pm 1.6$ & $<0.001 *$ \\
\hline \multirow[t]{4}{*}{ COP Velocity $(\mathrm{cm} / \mathrm{s})$ Eye Closed } & Immediate & $40.2 \pm 1.7$ & $43.7 \pm 1.9$ & \\
\hline & 3 min After & $40.1 \pm 1.8$ & $45.8 \pm 1, z$ & \\
\hline & $6 \mathrm{~min}$ After & $36.9 \pm 1.7$ & $43.9 \pm 1.8$ & \\
\hline & $9 \mathrm{~min}$ After & $37.4 \pm 1.8$ & $41.4 \pm 1.8$ & \\
\hline
\end{tabular}


Table 3. The Comparison of total area and velocity between athlete and non-athlete groups before, immediately, $3 \mathrm{~min}, 6 \mathrm{~min}$ and $9 \mathrm{~min}$ after fatigue (Independent T-test)

\begin{tabular}{lcccc}
\hline Variables & & Athlete & Non-athlete & $\mathrm{p}^{*}$ \\
\hline COP displacement & Before & $0.247 \pm 0.019$ & $0.24 \pm 0.013$ & 0.91 \\
$(\mathrm{~cm})$ & Immediate & $0.28 \pm 0.018$ & $0.30 \pm 0.014$ & $0.001^{*}$ \\
Eye Closed & 3min After & $0.24 \pm 0.019$ & $0.33 \pm 0.017$ & $<0.001^{*}$ \\
& 6min After & $0.24 \pm 0.019$ & $0.25 \pm 0.018$ & 0.13 \\
& 9min After & $0.23 \pm 0.013$ & $0.24 \pm 0.016$ & 0.34 \\
COP Velocity & Before & $38.13 \pm 1.8$ & $42.2 \pm 1.6$ & $<0.001^{*}$ \\
$(\mathrm{~cm} / \mathrm{s})$ & Immediate & $40.2 \pm 1.7$ & $43.7 \pm 1.9$ & $<0.001^{*}$ \\
Eye Closed & 3min After & $40.1 \pm 1.8$ & $45.8 \pm 1, \boldsymbol{4}$ & $<0.001^{*}$ \\
& 6min After & $36.9 \pm 1.7$ & $43.9 \pm 1.8$ & $<0.001^{*}$ \\
& 9min After & $37.4 \pm 1.8$ & $41.4 \pm 1.8$ & $<0.001^{*}$ \\
\hline
\end{tabular}

Moreover, velocity with eyes closed significantly decreased in the intervention group compared to the control group immediately, 3 minutes, 6 minutes, and 9 minutes after fatigue $(\mathrm{p}<0.001)$ (Table 3$)$.

\section{Discussion}

The difference in the type of the fatigued muscles and type of fatigue protocol has caused the studies to represent different results in the remained duration of fatigue effect. Some researchers used general exercises to produce naturally central fatigue (14). Other researchers fatigued 1 muscle or a specific group of muscles that caused more peripheral fatigue (16). In previous studies, it had been stated if the maximum voluntary contraction reduces about $27 \%$, the mean velocity will increase for 15 minutes (21). Yaggie et al. found that $50 \%$ reduction of the maximum voluntarily contraction power in changing amplitude would be increased for 20 minutes (12). On the other hand, Dickin et al. concluded that postural control disorders with $30 \%$ reduction of the maximum voluntarily contraction will remain for 30 minutes (21). According to the present study, duration of isometric fatigue effect $(50 \%$ reduction of the maximum voluntarily contraction) on the total area lasted for 3 minutes in athletes. The existence of difference in the type of fatigue protocol was the possible justification of different durations of fatigue effect between these studies. In addition to the type of exercise, the amount of reduction in the maximum voluntarily contraction power influenced the duration of fatigue effect on postural control variables. Harkins et al. found the same results. They revealed that the duration of postural control disorder with $70 \%$ reduction in the maximum voluntarily contraction was about $75 \mathrm{sec}-$ onds, and they further indicated that this time decreased to 35 seconds for $50 \%$ reduction (13).

Moreover, duration of postural control disturbance in the study of Harkins et al. was less than the current study, which might be due to the location of the muscle fatigued. It was found that fatigue in the lumbar extensor muscles included more postural control disorder than the fatigue of other muscles such as ankle muscles (22). In another study conducted by Dickin et al. (23), it was found that the duration to return to the initial state after fatigue in ankle muscles was faster than muscles' fatigue around the knee. Perhaps, the different characteristics of fiber in muscles could be mentioned in the participants of these studies (different types of fiber muscle). Duration to return to the baseline (pre- fatigue) in electromyography parameters of muscles was more than returning to the initial state of muscles' power (24). Perhaps, the type of muscle contraction such as the concentric or eccentric influenced the duration to return to the in baseline. Central nervous system might also work to enhance motor functional abilities. In the study conducted by Sahlin et al. knee extensor muscles power recovered to the pre- fatigue until 4 minutes after fatigue (16). In the present study, total area was recovered for 3 minutes after fatigue in athlete participants. These findings are logical because it has been found that the duration to return to the baseline postural sway was less than the duration to return to the baseline of muscles' contraction power (25).

\section{Conclusion}

Lumbar extensors fatigue significantly changed mean velocity and total area in soccer player participants. Lumbar fatigue had more influences on COP distance than the mean velocity in the 2 groups. Moreover, it was found that soccer players could recover their balance faster than non-athlete participants.

\section{Conflict of Interests}

The authors declare that they have no competing interests.

\section{References}

1. Shumway-Cook A, Woollacott MH. Motor control : translating research into clinical practice. Philadelphia: Wolters Kluwer Health/Lippincott Williams \& Wilkins; 2012

2. Era P, Sainio P, Koskinen S, Haavisto P, Vaara M, Aromaa A. Postural balance in a random sample of 7,979 subjects aged 30 years and over. Gerontology. 2006;52(4):204-13.

3. Tsai LC, Yu B, Mercer VS, Gross MT. Comparison of different structural foot types for measures of standing postural control. Journal of Orthopaedic \& Sports Physical Therapy. 2006;36(12):942-53.

4. Madigan ML, Davidson BS, Nussbaum MA. Postural sway and joint kinematics during quiet standing are affected by lumbar extensor fatigue. Hum Mov Sci. 2006 Dec;25(6):788-99.

5. Powers SK, Jackson MJ. Exercise-induced oxidative stress: cellular mechanisms and impact on muscle force production. Physiol Rev. 2008;88(4):1243-76.

6. Rampinini E, Bosio A, Ferraresi I, Petruolo A, Morelli A, Sassi A. Match-related fatigue in soccer players. Med Sci Sports Exerc. 2011 Nov;43(11):2161-70. PubMed PMID: 21502891.

7. Pau M, Ibba G, Attene G. Fatigue-induced balance impairment in young soccer players. J Athl Train. 2014 Jul-Aug;49(4):454-61.

8. Arliani GG, Almeida GP, Dos Santos CV, Venturini AM, Astur Dda C, Cohen M. The effects of exertion on the postural stability in young soccer players. Acta ortopedica brasileira. 2013 May;21(3):155-8.

9. Ishizuka T, Hess RA, Reuter B, Federico MS, Yamada Y. Recovery of time on limits of stability from functional fatigue in Division II collegiate athletes. Journal of strength and conditioning research / National Strength \& Conditioning Association. 2011 Jul;25(7):1905-10. 10. Lin D, Seol H, Nussbaum MA, Madigan ML. Reliability of COP- 
based postural sway measures and age-related differences. Gait Posture. 2008;28(2):337-42.

11. Papa EV, Garg H, Dibble LE. Acute effects of muscle fatigue on anticipatory and reactive postural control in older individuals: a systematic review of the evidence. J Geriatr Phys Ther.2001; 2015 JanMar;38(1):40-8.

12. Yaggie JA, McGregor SJ. Effects of isokinetic ankle fatigue on the maintenance of balance and postural limits. Arch Phys Med Rehab. $2006 \mathrm{Feb} ; 83(2): 224-8$

13. Harkins KM, Mattacola CG, Uhl TL, Malone TR, McCrory JL. Effects of 2 ankle fatigue models on the duration of postural stability dysfunction. J Athl Train. 2006;40(3):191-4.

14. Bove M, Faelli E, Tacchino A, Lofrano F, Cogo CE, Ruggeri P. Postural control after a strenuous treadmill exercise. Neuroscience letters. 2007;418(3):276-81.

15. Erkmen N, Taskin H, Kaplan T, Sanioglu A. Balance performance and recovery after exercise with water intake, sport drink intake and no fluid. J Exer Sci Fit. 2010;8(2):105-12.

16. Sahlin K, Ren JM. Relationship of contraction capacity to metabolic changes during recovery from a fatiguing contraction. J App Physiol (Bethesda, Md : 2005). 2006 Aug;67(2):648-54.

17. Allum JH, Bloem BR, Carpenter MG, Hulliger M, Hadders-Algra M. Proprioceptive control of posture: a review of new concepts. Gait Posture. 2007;8(3):214-42.

18. Olson MW. Trunk extensor fatigue influences trunk muscle activities during walking gait. J Electromyograph Kinesiol. 2010;20(1):17-24.

19. Bensoussan L, Viton J-M, Schieppati M, Collado H, de Bovis VM, Mesure S, et al. Changes in postural control in hemiplegic patients after stroke performing a dual task. Arch Phys Med Rehab. 2007;88(8):100915.

20. Błaszczyk J, Orawiec R, Duda-Kłodowska D, Opala G. Assessment of postural instability in patients with Parkinson's disease. Exper Brain Res. 2007;183(1):107-14.

21. Pline KM, Madigan ML, Nussbaum MA. Influence of fatigue time and level on increases in postural sway. Ergonomics. 2006 Dec $15 ; 49(15): 1639-48$.

22. Lin D, Nussbaum MA, Seol H, Singh NB, Madigan ML, Wojcik LA. Acute effects of localized muscle fatigue on postural control and patterns of recovery during upright stance: influence of fatigue location and age. Eur J Appl Physiol. 2009;106(3):425-34.

23. Dickin DC, Doan JB. Postural stability in altered and unaltered sensory environments following fatiguing exercise of lower extremity joints. Scand J Med Sci Sport. 2008;18(6):765-72.

24. Walsh M, Peper A, Bierbaum S, Karamanidis K, Arampatzis A. Effects of submaximal fatiguing contractions on the components of dynamic stability control after forward falls. J Electromyograph Kinesiol. 2011;21(2):270-5.

25. Paillard T, Margnes E, Maitre J, Chaubet V, Francois Y, Jully JL, et al. Electrical stimulation superimposed onto voluntary muscular contraction reduces deterioration of both postural control and quadriceps femoris muscle strength. Neuroscience. 2010;165(4):1471- 